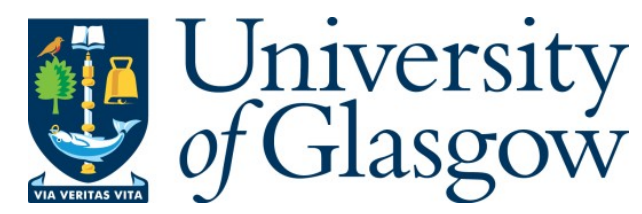

Hild, S., Leavey, S., Graf, C., and Sorazu, B. (2014) Smart charging technologies for portable electronic devices. IEEE Transactions on Smart Grid, 5 (1). pp. 328-336. ISSN 1949-3053

Copyright $\odot 2014$ IEEE

A copy can be downloaded for personal non-commercial research or study, without prior permission or charge

Content must not be changed in any way or reproduced in any format or medium without the formal permission of the copyright holder(s)

When referring to this work, full bibliographic details must be given

http://eprints.gla.ac.uk/90897/

Deposited on: 20 February 2014

Enlighten - Research publications by members of the University of Glasgow http://eprints.gla.ac.uk 


\title{
Smart Charging Technologies for Portable Electronic Devices
}

\author{
Stefan Hild, Sean Leavey, Christian Gräf and Borja Sorazu
}

\begin{abstract}
In this article we describe our efforts of extending demand-side control concepts to the application in portable electronic devices, such as laptop computers, mobile phones and tablet computers. As these devices feature built-in energy storage (in the form of batteries) and the ability to run complex control routines, they are well-suited for the implementation of smart charging concepts. We developed simple hardware and software based prototypes of smart charging controllers for a laptop computer that steer the charging process depending on the frequency of the electricity grid and in case of the software implementation also based on the battery charge status. If similar techniques are incorporated into millions of devices in UK households, this can contribute significantly to the stability of the electricity grid, help to mitigate the short-term power production fluctuations from renewable energy sources and avoid the high cost of building and maintaining conventional power plants as standby reserve.
\end{abstract}

Index Terms-Demand Side Control, Smart Grids, Smart Charging

\section{INTRODUCTION}

In the recent past, many countries have strengthened their efforts to increase the fraction of electricity produced from renewable energy sources in order to reduce the greenhouse gas emissions from fossil fuel power plants. For instance, in the Climate Change Act (2008), the UK government set out targets to cut $\mathrm{CO}_{2}$ equivalent emissions by $34 \%$ compared to 1990 levels by 2020 [1]. Additionally, in Scotland, the devolved government has been considering plans "to meet an equivalent of $100 \%$ demand for electricity from renewable energy by 2020" [2]. The European Union also has a directive which legislates that the UK should increase production of electricity from renewable energy sources by $15 \%$ over the same period [3]. Such policies are leading to an increase in the proportion of renewable energy sources as a means of electricity supply.

The current electricity grid of the UK, similar to all other countries in the world, does not provide any significant storage capacity. The consequence is that each kilowatt-hour of electricity that we want to use somewhere within the country must be produced instantaneously at the very same moment, i.e. every second our electricity production needs to be matched exactly to our electricity demand.

This requirement is probably the most demanding challenge towards the realization of a sustainable energy supply based

S. Hild, S. Leavey and B. Sorazu are with the School of Physics and Astronomy at the University of Glasgow, G12 8QQ, United Kingdom

C. Gräf is with the Experimental Division, Albert-Einstein-Institut (MaxPlanck-Institut für Gravitationsphysik), 30167 Hannover, Germany.

Manuscript received September 25, 2012; on renewable energy sources such as photovoltaic and wind power, because their electricity output varies strongly with time, is often not reliably predictable and at most times differs greatly from the actual electricity demand. In particular, the lack of available renewable energy during prolonged calm and cloudy periods poses a major challenge. To provide enough electricity on such days one can either resort to a large fleet of conventional power plants in standby mode, ready to take over when the renewable energy sources cannot satisfy the demand, or build huge facilities (such as pumped storage or batteries) for long duration electricity storage. Both of these approaches require costly hardware to be built and maintained on a scale similar to the existing fleet of conventional power plants in the UK.

Smart grids and so-called demand control techniques [4], [5], [6] provide a less costly and less hardware extensive alternative. This is achieved by relaxing the link between electricity production and demand, based on introducing a mechanism such that the demand can be controlled to match the variations in supply. This can be done by shifting less urgently required electricity consumption out of times of high demand into periods of surplus energy production. For instance, if a person were to connect their electric car or a laptop to a charger in the evening, it would probably not matter to the individual exactly when during the night it is charged, as long as it is fully charged in the morning. The development and introduction of smart charging technologies into as many areas of our daily life as possible is the most promising solution for a reliable integration of renewable energy sources into the UK energy portfolio. Ultimately, increasing the deployment of smart charging technology will allow us to reduce the number of required standby power plants and to save the associated costs for construction and operation.

There is ongoing research on the use of demand control techniques in consumer hardware. Short et al. [4] have demonstrated a demand controlled domestic refrigerator, showing that the technique is useful in reducing the need for standby generation capacity when deployed on a large scale for both short supply or demand transients and prolonged fluctuations caused by varying output from wind power. On a commercial scale, the UK company Open Energi have recently started equipping the supermarket chain Sainsburys' refrigeration portfolio with demand control technology, in the hope of saving 100,000 tons of $\mathrm{CO}_{2}$ equivalent emissions over 10 years [7].

There is also interest in incorporating the technology into electric vehicles [8] and plug-in hybrid electric vehicles [9],[10]. Huang et al. [12] describe an inductive power transfer mechanism for charging electric cars which responds to grid 
frequency instabilities caused by an emulated wind turbine. The system was shown to have improved frequency stability significantly, though the scope was limited to a single load on a micro-grid. Work by Ifland et al. [13] outlines a theory considering the use of electric vehicles as demand control devices, shaping their power load during charging to coincide with periods of high supply. This is achieved through the use of power line communication and electricity market pricing mechanisms. Similarly, Bashash et al. have developed a plugin hybrid vehicle smart charger which optimises the charging process to the current electricity price and shapes load to increase battery longevity [11].

In this article we extend the application of demand control concepts to portable electronic devices, such as laptop computers, mobile phones and tablet computers. We will describe the general principle of how to realize smart charging portable electronic devices in the next section. In Sections III and IV we discuss hardware prototypes of smart charging laptops, i.e. simple external on-off controllers for laptop power supplies, similar to concepts that have been used for controlling electric appliances [14],[15]. As we will discuss in Section V, in a second step we also realised a smart charging laptop that is entirely based on software, which controls the charging process by supervising the battery management control system. Future developments including the application of more complex charging algorithms are discussed in Section VI. Finally we give a conclusion in Section VII.

\section{THE CONCEPT OF SMART CHARGING OF PORTABLE ELECTRONIC DEVICES}

\section{A. The principle}

Portable electronic devices, such as laptop computers, mobile phones and tablet computers ${ }^{1}$ can easily be transformed to "be smart" in terms of energy handling. First of all, without any additional hardware or cost they already feature built-in electricity storage (the battery). Additionally, these devices are inherently capable of performing complex algorithms, required for smart charging, as well as communicating with other devices, for instance via the Internet to receive or exchange information on the status of the electricity grid.

So, imagine the following scenario: in a few years from now we can all choose in the power management settings of our laptops not only options for long battery life or high laptop performance, but also an option which can be set to smart charging in order to mitigate power fluctuations from time-varying renewable energy sources. This could be incentivized either through social responsibility or by power companies which could reward customers for using the setting by monitoring energy consumption with in-home smart meters.

How would this work in principle? First of all, the laptop would need to know whether there is a deficit or a surplus in the electricity grid. This can either be obtained from a central server via the Internet (or from a server controlled by the user's power company), or deduced from a local measurement of the

\footnotetext{
${ }^{1}$ In the following we will always use a laptop computer as our example, but similar technologies can also be applied to tablets, phones and other portable electronic devices.
}

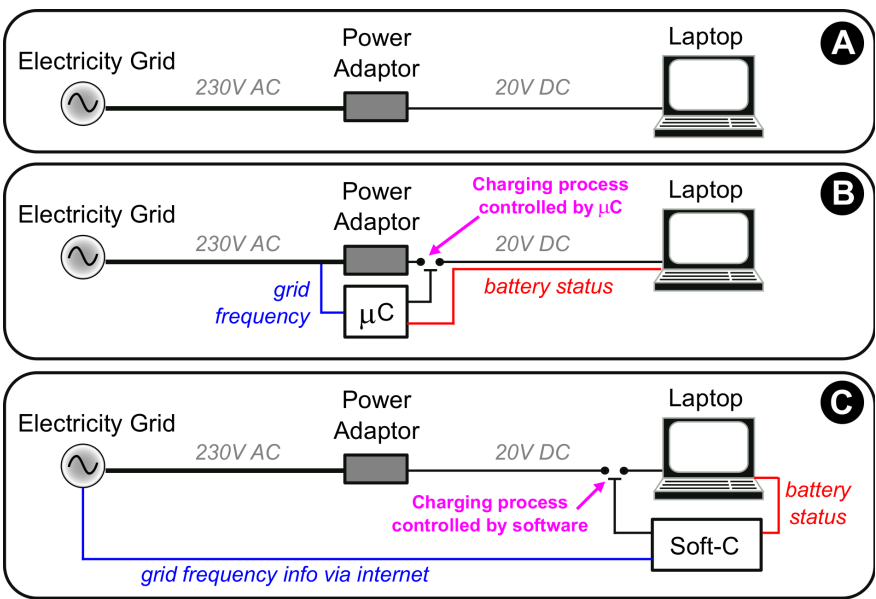

Fig. 1. Sketch illustrating the principle of smart charging laptops. A) a standard laptop. B) A microcontroller $(\mu \mathrm{C})$ performs a local measurement of the grid frequency and receives the battery charge state information from the laptop. On the basis of these two pieces of information an algorithm in the microcontroller controls the charging process. (Instead of the local frequency measurement the grid frequency can also be communicated to the microcontroller by means of a higher harmonic modulation of the grid frequency itself). C) The charging process is guided by a software controller (Soft-C) inside the laptop using the battery charge state information as well as the grid frequency information which could be received from a central server via the Internet.

grid frequency, itself an indicator of the electricity supply-todemand ratio in the national grid [4]. A grid frequency above $50 \mathrm{~Hz}$ indicates a surplus of electricity in the grid, whereas a grid frequency below $50 \mathrm{~Hz}$ indicates that the electricity demand outweighs the electricity supply. ${ }^{2}$ Another way to transfer the information of the electricity grid from a central location to individual consumers would be usage of high frequency amplitude modulations of the grid voltage, similar to as is done to control night storage heating tariffs or the switching of street lamps. Then depending on whether there is too much or too little electricity in the grid and also taking the current battery charge status and the user's habits into account, a controller could steer the charging of the laptop in a smart way. Such a controller can either be implemented in hardware inside the power adaptor or the laptop itself, or it could simply be a software algorithm steering the charging of the battery. As we will discuss in more detail in Section VI, when it comes to electronic devices, different individuals have different personal user habits, and therefore personalized charging profiles are bound to vary from case to case. For instance, a particular user might insist upon a minimum level of charge that should always be available, while others may require a fully charged laptop at a particular time of day (such as before a morning train journey).

Figure 1 gives a simplified overview of different principles for smart charging of portable electronic devices. The schematic drawing in the top frame of Figure 1, denoted as A, illustrates the charging of a standard laptop. A power adaptor is connected to the electricity grid and converts the $230 \mathrm{~V} \mathrm{AC}$ into about $15-20 \mathrm{~V}$ DC (the exact value varies for different

\footnotetext{
${ }^{2}$ Please note that throughout this article we refer to the grid frequency as $50 \mathrm{~Hz}$. However, the same techniques described here can be easily transferred to electricity grids using a frequency of $60 \mathrm{~Hz}$.
} 
devices and manufacturers). The DC voltage is connected to the laptop and depending on the battery status the charging is switched on or off within the laptop itself.

The sketch in the middle frame of Figure 1, labeled B, depicts a setup in which a simple external on-off controller is used to control the DC power supply connection between the power adaptor and the laptop. In this example the controller unit is implemented in hardware, for instance by analog electronics or a microcontroller $(\mu \mathrm{C})$. Such a microcontroller can be used for measuring the grid frequency, to deduce the current supply to demand ratio of the electricty grid (see Section IV for details of an example implementation). Ideally, the microcontroller has knowledge of the actual charge status of the laptop battery. This information, the grid frequency and the charge status of the laptop battery, can be sent to software running on the microcontroller to generate a signal, which can then be used to control the smart charging process, e.g. by toggling an electronic switch.

A cheaper alternative to the hardware implementation of a smart charging laptop can be realized purely in software (see plot $\mathrm{C}$ of Figure 1). As most portable electronic devices are already now connected to the Internet for a major fraction of their time in use (and it seems likely that in the future the fraction will only increase), it can be more efficient to perform the grid frequency measurement centrally and send the information via the Internet to millions of devices. This option would not only save on costs related to hardware, but also allow the implementation of a measurement setup providing a much higher precision than is feasible in the lowcost setup included in every power adaptor or laptop. The grid status information received from a central server can then again be combined with the battery charge status and the personal user preferences and used as input for an algorithm that controls the charging process.

Obviously, the scenarios described above illustrate only two examples of a range of possible implementations. Combinations of various elements of the concepts shown in Figure 1 B and $\mathrm{C}$ are possible. In Sections III and IV we present and discuss first hardware prototypes of smart charging laptops, while in Section V we present a software based implementation of a smart laptop charger that we realized.

\section{B. Smart charging and battery health}

If the smart charging concept described above is applied to portable electronic devices then the characteristics of the charge and discharge cycles may change slightly. When a normal laptop computer is connected to its power supply it charges the battery continuously up to full charge and then trickles. In contrast when connecting a smart charging laptop system to the electricity grid it would usually not fully charge the battery in a single go, but rather there might be some interruptions in the charging during periods in which the electricity demand in the grid outweighs the available supply. In addition the integrated duration of being in trickle mode will be reduced.

Both of these differences originating from the application of smart charging would not have any serious negative effects on the health of Lithium batteries. First of all, studies have shown that there is no significant correlation between the depth of discharge (DOD) and the battery degradation, but only a correlation between total processed capacity of the battery and the battery degradation [16]. As the smart charging concept would only change the DOD, not the total processed capacity of the battery, we do not expect any negative effects. Moreover, the non-continuous charging when using a smart controller would probably lead to a reduced peak temperature during the charging process, which would actually reduce the battery degradation [17].

\section{Benefits of smart charging of portable electronic devices}

Obviously, due to their significantly smaller capacity, smart charging of portable electronic devices has in the long term a lower potential of demand to supply balancing than other currently discussed approaches such as smart charging of electric cars. However, in the short and medium term future the number of existing portable electronic devices is orders of magnitude larger than the number of existing electric cars. Also, as laptop computers and other portable electronic devices anyway always have a rechargeable battery, the smart charging concept can be integrated in future hardware without any significant cost.

Let us assume that $2 / 3$ of the UK population were to use a laptop (or similar device) and half of these laptops would be connected to a charger at a time. Then 20 million devices would be connected to the National Grid at any given time. If we further assume that these devices would charge on average with a power of $50 \mathrm{~W}$ each, then if optimally deployed the smart charging concept for laptops alone would allow us to avoid building and operating on standby about one large power plant of the $1 \mathrm{GW}$ class and save the associated costs. A more comprehensive analysis of the large scale effect of our concept onto the electricity grid is currently under way and will be published in a separate article.

Moreover, this technology will allow each individual laptop user to save money, as it is expected that in future the price for electricity will not be constant, but vary depending on the actual demand and availability of electricity [6].

\section{PROTOTYPE OF A SMART CHARGING LAPTOP BASED ON AN EXTERNAL ON-OFF CONTROLLER FOR THE LAPTOP POWER SUPPLY}

For the first simplified prototype of a smart charging laptop we used a combination of simple analog electronics and a real time Linux digital control system [18], which is available in our lab. Figure 2 shows a schematic of the setup. This prototype measures whether the grid frequency is above a certain threshold and if so it switches on the charging of the laptop.

An AC adaptor (transformer) is used to to obtain a pick off voltage of $5 \mathrm{~V} \mathrm{AC}$. This signal is first low pass filtered to prevent aliasing and then digitized with an analog-todigital converter (ADC) at a sampling frequency of $20 \mathrm{kHz}$. Next we remove higher harmonic components as well as any unwanted DC components by filtering with a bandpass from 

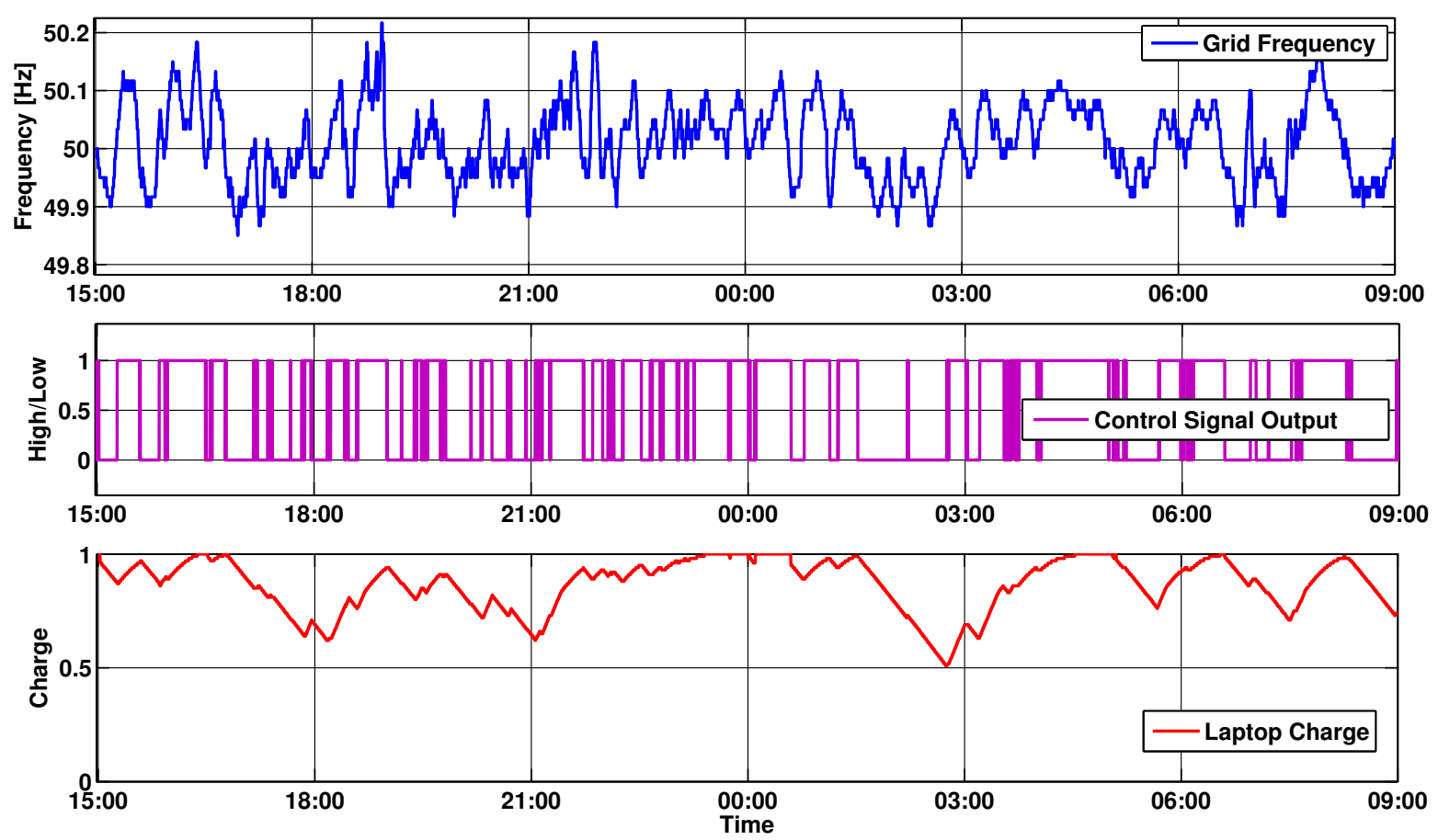

Fig. 3. Smart charging of a laptop using the setup shown in Figure 2. The upper plot shows the locally measured grid frequency for a period of 18 hours. The center plot shows the output signal of the smart charging controller, whilst the bottom plot shows the charge status of the laptop, where 1.0 and 0 correspond to $100 \%$ and $0 \%$ charge, respectively. It can clearly be seen that the battery charge decreases during periods when the grid frequency is below $50 \mathrm{~Hz}$ and the laptop runs on its battery, while it recharges the battery or trickles whenever the grid frequency is above $50 \mathrm{~Hz}$.

$45-55 \mathrm{~Hz}$. The resulting signal is split into two paths in order to perform a differential measurement using two bandpass filters (Butterworth, 6th order), one tuned to be slightly below the threshold frequency $(50.0 \mathrm{~Hz}$ in the setup shown in Figure 2 ) and the second one tuned to be slightly above the threshold frequency. If the actual grid frequency is above the threshold frequency, the amplitude after the bandpass stages will be larger in the right hand branch than in the left one. In order to get a DC measurement of the amplitude after the bandpass filter, we convert the signal into a symmetric rectangular voltage and take the absolute value. After low-passing, the signals from the two branches are subtracted from each other and passed through a second low pass filter. A comparator is used to set the output of the digital controller to high if the signal after the subtracter is smaller than 0 (i.e. if the frequency is above the threshold) and to low if the signal after the subtracter is greater than 0 (i.e. if the frequency is below the threshold). Finally, the digital signal is output via a digitalto-analog converter (DAC) and used to switch a relay, which can connect or disconnect the AC adaptor from the supply voltage.

Figure 3 shows the performance of this simple on-off prototype over a period of 18 hours and using a frequency threshold of $50.00 \mathrm{~Hz}$. The top trace shows the time series of the grid frequency, varying between about 49.85 and $50.20 \mathrm{~Hz}$. The output signal of the digital control system is shown in the center trace, with y-values of 1 representing active charging and $y$-values of 0 indicating that the charging is suspended. Finally the bottom trace shows the battery charge of the connected laptop (with charge level normalized between 0 and 1), a Dell Latitude 5520, which was connected to a power socket and in normal operation (playing music and keeping the screen lit continuously to simulate a typical workload) during the whole measurement time. When the grid frequency drops below $50 \mathrm{~Hz}$ the laptop suspends the charging process and runs on its battery and therefore the charge decreases. Whenever the frequency is above $50 \mathrm{~Hz}$ the charging is switched on again and the charge increases until the battery is fully charged. For this randomly chosen stretch of time the charge of the laptop varied between about $50 \%$ and $100 \%$.

The charge status of the laptop obviously depends not only on the evolution of the grid frequency, but also on the rates of charge and discharge. The slower the rate of discharge and the larger the capacity of the battery, the higher the average charge level of the battery. Similarly, the faster the rate of charge, the higher the average charge level. In addition to the Dell laptop (in the following referred to as Laptop 1), we also tested the smart charging controller with a MacBook Pro ${ }^{\circledR}$ laptop (in the following referred to as Laptop 2), which yielded a higher average charge value because of a higher ratio of rates of charge and discharge (see Figure 9 in Appendix A). In addition, the longer battery life of Laptop 2 made it possible for Laptop 2 to bridge even several-hour long stretches of the grid frequency being below $50 \mathrm{~Hz}$, whereas the battery of Laptop 1 would have been flat after about 2.5 hours (even when started from a fully charged battery).

One way to increase the average battery charge, especially for laptops such as Laptop 1 with a poor charge to discharge 


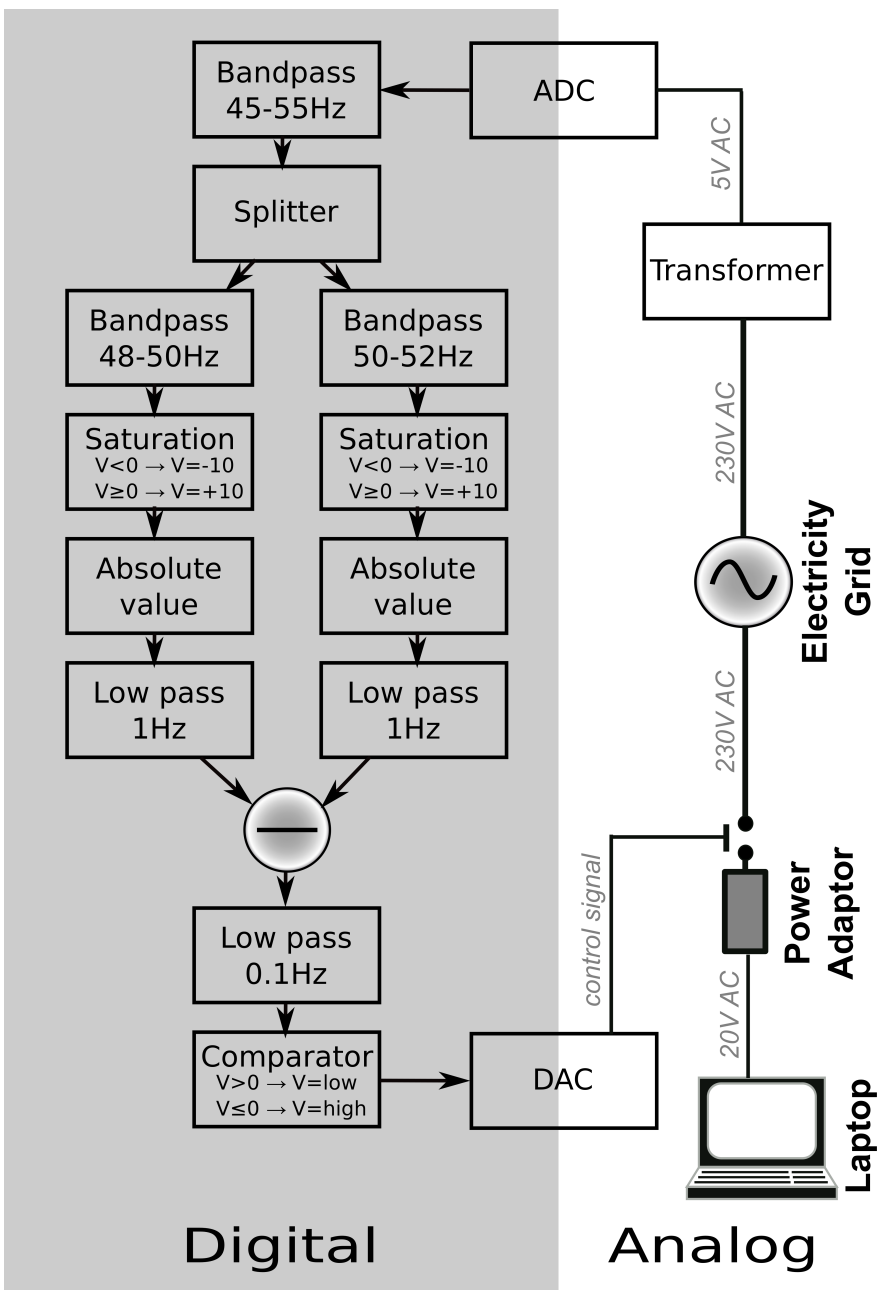

Fig. 2. Schematic of a simple external on-off implementation of a smart charging laptop. The grid frequency is analysed by a digital system using a differential bandpass technique (see a more detailed explanation in the text). If the grid frequency is above a certain frequency (for instance $50.00 \mathrm{~Hz}$ ) then the digital control system switches on the power to the AC adaptor of the laptop. If the grid frequency is below the set threshold, the charging of the laptop is suspended. (Used abbreviations: $\mathrm{ADC}=$ analog-to-digital converter, $\mathrm{DAC}=$ digital-to-analog converter.)

ratio or a degraded battery, is to reduce the frequency threshold to below $50 \mathrm{~Hz}$. Obviously this comes at the expense of reduced mitigation of grid fluctuations, as the laptop still charges even though the grid frequency has fallen slightly below $50 \mathrm{~Hz}$. However, in periods when the grid frequency is significantly below $50 \mathrm{~Hz}$, the smart charging laptop with a reduced threshold frequency still provides the full level of mitigation.

Using real frequency data from the UK grid, we developed simulations of the battery status of our Laptop 1 for various frequency thresholds. The result for a 21 day long dataset is shown in Figure 4. While for threshold frequencies of 49.995 and $49.985 \mathrm{~Hz}$ the Dell laptop still occasionally runs out of battery, lowering the threshold to $49.975 \mathrm{~Hz}$ seems to guarantee that the laptop charge always stays above $40 \%$.

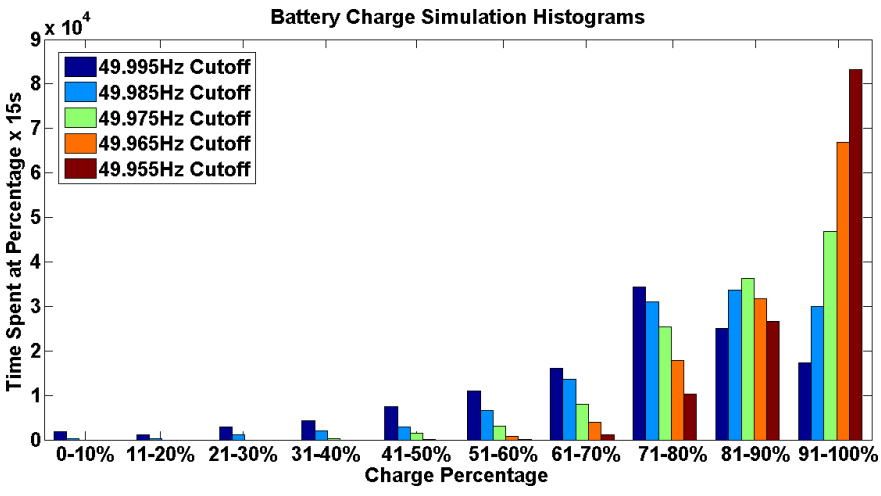

Fig. 4. Comparison of the charge status of our smart charging prototype connected to the Dell laptop (Laptop 1) for the application of different threshold frequencies. In order to guarantee comparability of the results, we developed a simulation of the battery charge that runs a virtual smart charging controller for different threshold frequencies over exactly the same grid frequency data set of 21 days duration. The lower the chosen frequency threshold, the higher the average charge of the laptop. While for threshold frequencies of 49.995 and $49.985 \mathrm{~Hz}$ Laptop 1 could occasionally run out of charge, for threshold frequencies of $49.975 \mathrm{~Hz}$ and below it would always keep at least a charge of larger than $30 \%$. Please note that these results are specific for Laptop 1 and should only be seen as an illustrating example. As shown in Appendix A, Laptop 2 would provide a significantly higher average battery charge and even for a threshold of $50.000 \mathrm{~Hz}$ would not usually run out of charge.

\section{MiNIATURIZATION OF OUR HARDWARE PROTOTYPE USING A MICROCONTROLLER}

Since the hardware prototype described in the previous section relied on a stationary digital control system, we then developed a miniaturized and portable version of a smart laptop charger. The simplified setup is shown in Figure 5. Instead of the digital control system, in this prototype an Arduino ${ }^{\circledR}[19]$ microcontroller sits at the heart of the setup, performing the frequency measurement and controlling the charging process.

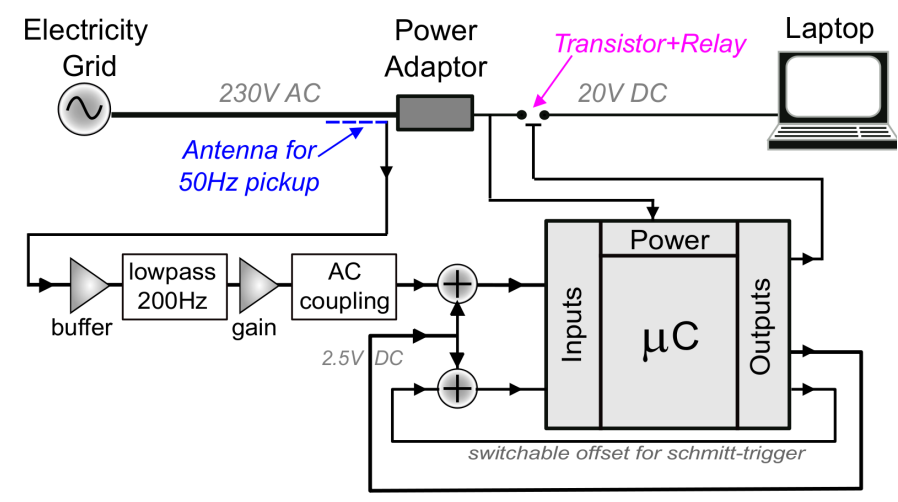

Fig. 5. Simplified schematic of miniaturized hardware prototype using a microcontroller. The grid frequency is first picked up by a small antenna, then cleaned and amplified by analog electronics and then measured with a microcontroller (see detailed description in the text). Depending on the actual grid frequency the mircrocontroller then gives out a control signal which is used to steer the charging process of the laptop. In order to guarantee the physical portability of the system, all electronic components and the microcontroller are powered from the laptop's power adaptor.

For the grid frequency measurement we used a short cable attached to the last few centimeters of the $230 \mathrm{~V}$ power cable. The signal picked up was further processed using simple 
analog electronics. First the signal is buffered, then passed through a low pass filter to remove any high-frequency noise, then it is amplified to about $1 \mathrm{~V}_{\mathrm{pp}}-2 \mathrm{~V}_{\mathrm{pp}}$ and finally $\mathrm{AC}$ coupled. As the inputs of the microcontroller are unipolar, we added an offset of $2.5 \mathrm{~V}$ onto the signal and input this signal into one $\mathrm{ADC}$ of the microcontroller and the offset without the signal into a second input.

The actual frequency measurement in the microcontroller is performed in the time domain [20]. A comparator compares the two input signals and triggers an interrupt whenever the difference of the two inputs changes sign. The frequency of the signal is then calculated by counting the clock cycles between consecutive interrupts. In order to increase the robustness of the frequency measurements against noise, a Schmitt trigger [21] is implemented by using one of the microcontrollers outputs to add a switchable offset onto the threshold voltage.
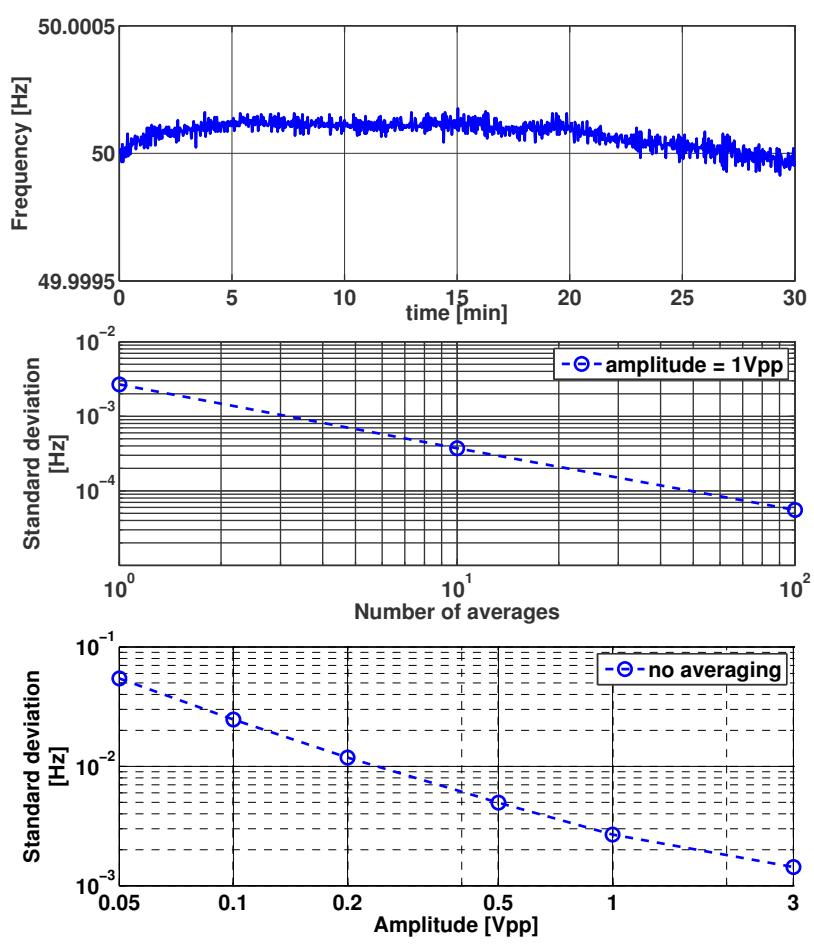

Fig. 6. Performance of the frequency measurement carried out with our microcontroller setup whilst connected to a fixed reference frequency of exactly $50 \mathrm{~Hz}$. Top plot: time series of a measurement with a signal amplitude of $1 \mathrm{Vpp}$ and 100 averages per measurement. Center plot: Dependence of the obtained frequency resolution on the number of averages. Using 100 averages a frequency resolution of $5 \times 10^{-5} \mathrm{~Hz}$ is achieved. Bottom plot: Dependence of the obtained frequency resolution on the signal amplitude.

In the microcontroller program one can set the number of sign changing events over which the algorithm should average for a single frequency measurement. If no averaging is used a frequency measurement is performed every 20 milliseconds. As shown in the center plot of Figure 6, without any averaging we obtain a frequency accuracy of about $2.5 \mathrm{mHz}$. Using 100 averages gives a frequency measurement every 2 seconds with a frequency accuracy of about $0.05 \mathrm{mHz}$, which should be more than sufficient for any smart charging application. The microcontroller can, aside from the grid frequency measurement, be programmed with code implementing more sophisticated smart charging techniques, i.e. in our simple prototype we used a simple logic that switches the charging on if the grid frequency is above one threshold and suspends the charging if it is below another threshold. These two threshold frequencies do not necessarily need to be identical; in actual fact it is beneficial to select slightly different thresholds for "debouncing", i.e. to avoid too frequent switching when the grid frequency is close to the thresholds. The charging control signal is then output by the microcontroller which controls via a transistor a relay in the circuit connecting the laptop and the power adaptor. In order to guarantee physical portability and flexibility of our system, the microcontroller and all anolog components are powered directly from the laptop's power adaptor. Currently our smart laptop charger fits into a box of similar dimensions to the power adaptor itself. Using SMD technology, it will be possible to reduce the size to a few cubic centimetres.

Figure 6 shows the performance of the frequency accuracy of the microcontroller setup. For these measurements the antenna was connected to a signal generator (Agilent 33120A) set to exactly $50 \mathrm{~Hz}$. As discussed earlier we found that for a given signal amplitude the frequency resolution improves significantly with the number of averages. Moreover, the frequency resolution also improves with the signal amplitude (for a fixed number of averages), as shown in the lowest subplot. This is due to the steeper slope of the signal during the zero-crossing of the comparator. The top plot of Figure 6 shows a timeseries of the frequency measurement using 100 averages and a signal amplitude of $1 \mathrm{~V} p$. We find the resolution to be much better than $0.1 \mathrm{mHz}$ and limited by a slow drift which is probably caused by a temperature driven perturbance of either the microcontroller's clock signal or the frequency stability of the signal generator itself [22]. However, the magnitude of this drift is too small to be of any concern for any potential application of smart charging concepts.

When comparing our two hardware prototypes it has to be noted that the concept based on the microcontroller has strong advantages over the realization using the setup described in Section III. The microcontroller setup is much smaller and therefore portable. Also it requires much less and much cheaper hardware. Furthermore it is of big advantage to the microcontroller setup that it has access to the absolute grid frequency as opposed to our implementation of a smart charger using the digital control system which is only able to detect whether the grid frequency is above or below the set threshold.

\section{SOFTWARE BASED REALISATION OF SMART CHARGING}

As discussed in Section II smart charging of portable electronic devices can also be realised entirely in software. Using a Levono Thinkpad T61 running the Ubuntu operating system we demonstrated the concept of a smart software charger which supervises the laptop's battery management system (BMS). The software accesses the current grid frequency via the Internet, for example from resources such as FNET [23], and reads in the battery charge status. The availability of the battery charge status allowed us to introduce a minimal charge 
level of the battery that is never surpassed as long as the laptop is connected to its power supply. Only above this minimal charge threshold the grid frequency is used to suspend or enable the charging of the battery.

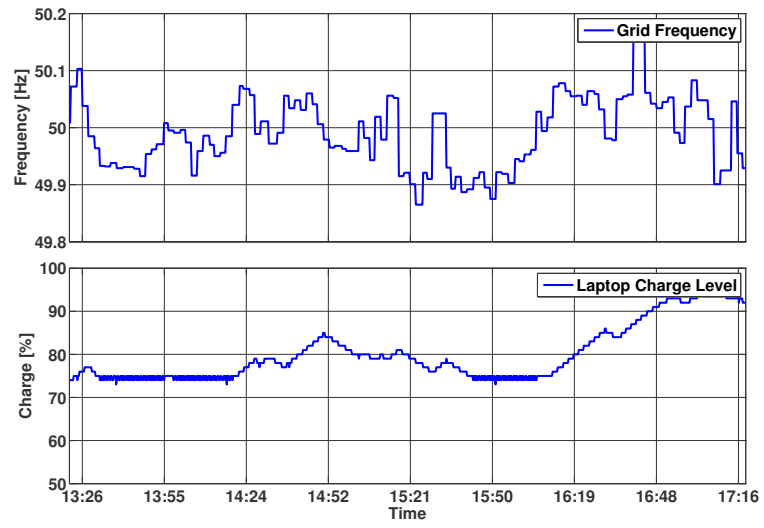

Fig. 7. Performance of a software based smart charger. Our software which supervises the battery management system (BMS) controls the charging process depending on the grid frequency (received via the Internet) and the actual battery charge level. If the battery charge falls below a threshold defined in the software ( $75 \%$ in this example), then the battery will be charged, even when the grid frequency is below $50 \mathrm{~Hz}$.

Figure 7 shows the performance of our software based charger using a minimal battery charge threshold of $75 \%$. In case the grid frequency is below $50 \mathrm{~Hz}$ and the battery charge is at the minimal battery charge threshold, this implementation causes the charging process to be suspended and resumed in quick succession. A way to avoid this very frequent switching of the charging we implemented in a further step a twothreshold concept as displayed in Figure 8. We used a lower threshold of $75 \%$ and a higher threshold of $80 \%$. When the battery charge level drops below $75 \%$ the battery is charged independently of the grid frequency. Only when the battery charge reaches the upper threshold at $80 \%$ will the charging controller start to take into account the grid frequency again. As a result, during the two times indicated by the vertical arrows the laptop battery is charged even though the grid frequency is below $50 \mathrm{~Hz}$. However, in contrast to Figure 7 employing the double threshold method, the previously observed continuous switching on and off of the battery charging is avoided.

The concept described in this section, which does not only take the grid frequency into account, but includes the actual battery charge level and steers the charging process via supervising the BMS, advances smart charging of portable electric devices beyond the external on-off controller for the power supply.

One clear disadvantage of the software based smart charger described here is the fact that it requires the laptop to have access to the Internet and therefore the laptop needs to be running when the smart charging modus should be active.

\section{Future DeVelopments}

The two hardware prototypes for a simple on-off smart charging controller as discussed in sections III and IV together

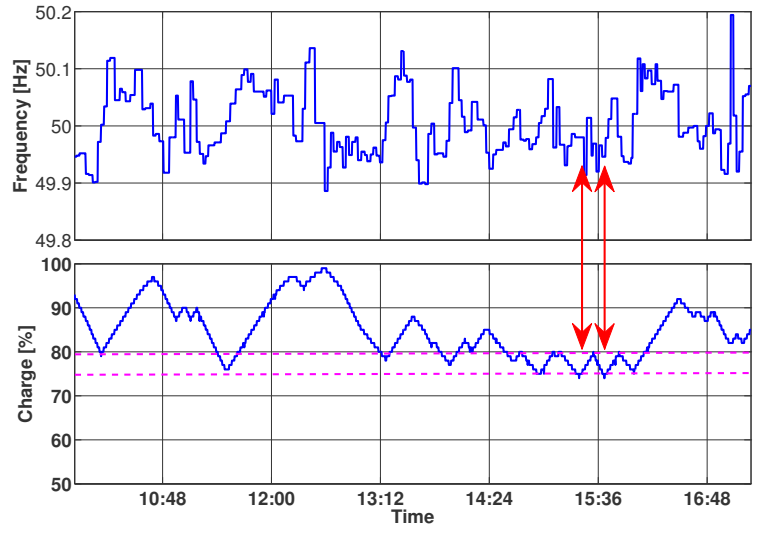

Fig. 8. Performance of a software based smart charger employing a two threshold method to avoid quick successions of switching on and off the charging process as it has been observed in Figure 7. The two thresholds (pink dashed lines) are set to $75 \%$ and $80 \%$ in this example.

with the software based smart charging concept described in the previous section constitute a first but nonetheless import step towards the practical realization of our smart charging concept.

In order to utilise the full potential of the concepts described in Section II it will be essential that the smart controller also has access to habits of the actual user.

Having access to the grid frequency, the battery charge level and the individual user profile/preferences would allow the implementation of more complex control algorithms which could include individual or combinations of the following features:

- A certain available charge status at particular user-defined times, such as to have the battery always hold at least $80 \%$ charge as of 08:00 on weekdays.

- Instead of using a 'hard' frequency threshold, a weighting factor could be introduced that takes into account not only of whether the grid frequency is above or a below the threshold, but also by how much the frequency is above or below the threshold. Assuming that the deviation of the grid frequency from $50 \mathrm{~Hz}$ is proportional to the supply-to-demand ratio, it will be possible to trade off the urgency of required demand suspension versus the user's urgency to charge their electronic devices on a particular occasion.

- Finally, it is also possibly desirable to investigate smart charging algorithms based on advanced control concepts, such as adaptive filters (for instance Wiener filters [24]) or self-learning systems, such as neural networks that can be optimised for the user's particular habits as well as for the characteristic demand evolution on daily, weekly and seasonal time scales.

At the moment it is not clear whether it will be best to have the complete smart charging algorithm entirely implemented into individual portable electronic devices, or whether it will be more efficient to have certain parts of the algorithm which are common for all users inside the electricity grid (such as the measurement of the grid frequency) performed centrally with 
the resulting information and/or steering signals being sent to the individual charging devices.

Furthermore, future efforts will include a detailed analysis (in a similar manner as for example has been carried out in the context of vehicle-to-grid technology [25], [26], [27]) of the large-scale effect and benefits of our concept onto the electricity grid when employed in millions of devices. Also we plan to investigate the size and cost requirements of a hardware controller based on a purpose-built microchip which could be integrated directly into the power supply of portable electronic devices by manufacturers.

\section{CONCLUSION}

Demand side control techniques and smart grid concepts are currently under consideration or are in the process of being implemented for a wide range of applications. In this article we have reported on our efforts to extend these techniques to applications for portable electronic devices featuring internal energy storage, such as laptop computers, mobile phones and tablet computers. We believe that the concepts we have described in this article could be applied in the medium term future to the majority of the portable electronic devices produced. This would not only contribute to the stabilisation of national electricity grids, helping to mitigate the electricity production fluctuations from renewable energy sources and helping to avoid the building of costly standby or backup power plants, but would also allow the end user to save money, by predominantly using electricity for charging their electronic devices in periods when it is cheap.

We have developed hardware and software based prototypes of a smart charging concepts for laptop computers. To the best of the authors' knowledge this was the world's first demonstration of demand control techniques applied to laptop computers. The hardware prototypes continuously perform a local measurement of the grid frequency and switch on or suspend charging, depending on whether the grid frequency is above or below a pre-set threshold, respectively. While the first prototype made use of a stationary digital control system and applied digital filtering techniques to analyse the grid frequency, the second prototype is based on a microcontroller to reduce the size of the device and the frequency measurement is performed by counting sign changes of the signal.

Our tests have shown that the smart charging concepts for portable electronic devices we described work entirely satisfactory. Moreover we found that the actual performance strongly depends on the certain properties of the underlying electronic devices, such as the overall battery life time and the ratio of the rates of charge and discharge. For systems with low battery lifetime and a low ratio of rates of charge and discharge, it can be preferable to use a frequency threshold of a few tens of $\mathrm{mHz}$ below $50 \mathrm{~Hz}$ to make sure that the electronic devices never run out of charge.

In addition we also developed a software based smart charging algorithm that steers the charging process of a laptop by supervising the battery management system and switching the charging on and off depending on the grid frequency as well as the user-defined battery charge level thresholds.
Future developments include investigations into how best to combine the hardware and software approaches. We also intend to test more complex charging controllers which include the personal user habits as an additional input to the smart charging algorithms. Moreover, the application of advanced charging algorithms, based on for instance adaptive filters or neural networks, will be investigated.

\section{APPENDIX A}

CHARGING AND DISCHARGING CHARACTERISTICS OF THE LAPTOP COMPUTERS WE TESTED

As mentioned in Section III the overall performance of any smart charging technique for an electronic device strongly depends on its battery capacity and its rates of charge and discharge. We define the ratio of charge and discharge rates to be:

$$
R=\frac{\text { Rate of Charge }}{\text { Rate of Discharge }} .
$$

The charge and discharge rates as well as their ratio for the laptops tested can been found in Table I. The discharge rate

\begin{tabular}{|l|c|c|c|}
\hline Laptop & $R_{\text {Charge }}$ & $R_{\text {Discharge }}$ & $R$ \\
\hline Laptop 1 (Dell E5520) & 0.0213 & 0.0115 & 1.8522 \\
\hline Laptop 2 (MacBook Pro ${ }^{(R)}$ ) & 0.0128 & 0.0056 & 2.2857 \\
\hline
\end{tabular}

TABLE I

A LIST OF THE RATES (PERCENTAGE POINTS PER SECOND) OF CHARGE,

$R_{\text {Charge }}$, AND Discharge, $R_{\text {Discharge }}$, AND THE RATIO BETWEeN THE TWO.

indicates how long it would take the laptop to go from fully charged down to a flat battery during normal operation. This duration we define as battery life time, and it turns out to be only about 2.5 hours for Laptop 1, whereas Laptop 2 has a battery life time of about 5 hours. Therefore, when used in combination with a smart charging controller, Laptop 2 shows better performance during extended periods (of the order hours) with a grid frequency below the charging threshold. Moreover, due also to its higher value for the ratio $R$, Laptop 2 regains its battery charge about twice as quickly as Laptop 1 during periods when the grid frequency is above the threshold. The result of these performance differences between Laptop 1 and Laptop 2 can be seen in Figure 9 which indicates the battery charge evolution of both laptops for the same period of time. While for this particular stretch of time the charge of Laptop 1 decrease to values as low as $50 \%$, the battery charge of Laptop 2 always stays above $75 \%$.

\section{ACKNOWLEDGMENT}

The authors would like to thank Kenneth Strain, Andreas Weidner and Mark Kille for fruitful discussions. We are also grateful for financial support from the John Roberston Bequest as well as the College of Science and Engineering at the University of Glasgow. 

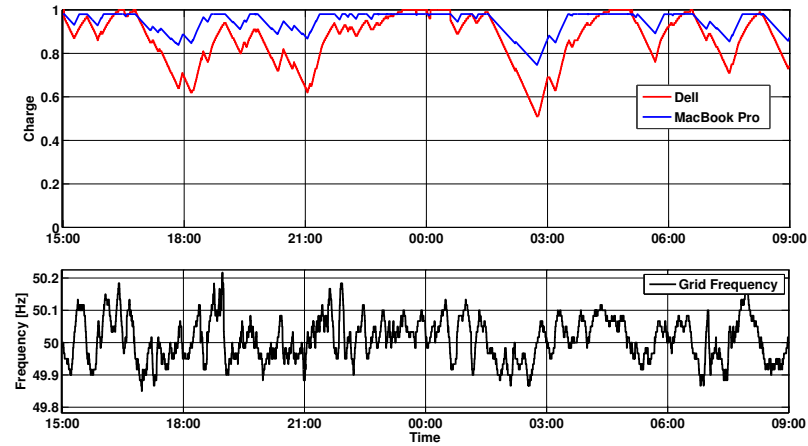

Fig. 9. Comparison of the measured charge status of Laptop 1 and the simulated charge status of Laptop 2 for the same time stretch as displayed in Figure 3. For both laptops a frequency threshold of exactly $50 \mathrm{~Hz}$ is used Due to its higher charge to discharge rate and its significantly larger battery lifetime, Laptop 2 would provide a significantly higher average battery charge than Laptop 1 when operated in a smart charging mode.

\section{REFERENCES}

[1] Parliament of the United Kingdom, Climate Change Act, 2008

[2] Scottish Government, 2020 Routemap for Renewable Energy in Scotland, available at http://www.scotland.gov.uk/Publications/2011/08/04110353/2

[3] The European Parliament, Directive 2009/28/EC, April 2009

[4] Joe A. Short, David G. Infield, and Leon L. Freris, Stabilization of Grid Frequency Through Dynamic Demand Control, IEEE Transactions on Power Systems Vol. 22, No. 3, August 2007, Page 1284.

[5] National Grid. Frequency Control by Demand Management (FCDM). http://www.nationalgrid.com/uk/Electricity/Balancing/services/ frequencyresponse/fcdm/, 2011

[6] Goran Strbac Demand side management: Benefits and challenges, Energy Policy Vol. 36 (2008) 4419-4426

[7] Open Energi, Case Study, available at http://www.openenergi.com/sainsburys (accessed 13/08/12).

[8] Francesco A. Amoroso and Gregorio Cappuccino: Impact of charging efficiency variations on the effectiveness of variable-rate-based charging strategies for electric vehicles, Journal of Power Sources, 196 (2011) 95749578

[9] Eric Sortomme and Mohamed El-Sharkawi: Optimal Charging Strategies for Unidirectional Vehicle-to-Grid, IEEE Trans. On smart grid, vol 2, 1, 2011

[10] Niklas Rotering and Marija Ilic: Optimal Charge Control of Plug-In Hybrid Electric Vehicles in Deregulated Electricity Markets, IEEE Trans. On Power System, vol 26, 3, 2011

[11] Saeid Bashash, Scott Moura, Joel Forman and Hosam Fathy: Plug-in hybrid electric vehicle charge pattern optimization for energy cost and battery longevity, Journal of Power Sources, 2010

[12] Chang-Yu Huang and Boys, J.T. and Covic, G.A. and Lee, J.R. and Stebbing, R.V., Vehicle Power and Propulsion Conference (VPPC), 2010 IEEE, Implementation and Evaluation of an IPT Battery Charging System in assisting Grid Frequency Stabilisation through Dynamic Demand Control. September 2010, pp. 1-6, DOI: 10.1109/VPPC.2010.5729207

[13] Ifland, M. and Exner, N. and Westermann, D., Energytech, 2011 IEEE, Appliance of Direct and Indirect Demand Side Management. May 2011, pp. 1-6, DOI: 10.1109/EnergyTech.2011.5948534

[14] David P. Chassin, Lynne Kiesling, Decentralized Coordination through Digital Technology, Dynamic Pricing, and Customer-Driven Control: The GridWise Testbed Demonstration Project, The Electricity Journal, Volume 21, Issue 8, October 2008, Pages 51-59.

[15] D.J. Hammerstrom et al: Pacific Northwest GridWise Testbed Demonstration Projects, Part II. Grid FriendlyTM Appliance Project. Available at http://www.pnl.gov/main/publications/external/technical_reports/PNNL17079.pdf

[16] Scott B. Peterson, Jay Apt and J.F. Whitacre: Lithium-ion battery cell degradation resulting from realistic vehicle and vehicle-to-grid utilization, Journal of Power Sources 195 (2010) 23852392.

[17] I. Bloom, B.W. Cole, J.J. Sohn, S.A. Jones, E.G. Polzin, V.S. Battaglia, G.L. Henriksen, C. Motloch, R. Richardson, T. Unkel- haeuser, D. Ingersoll, H.L. Case: An accelerated calendar and cycle life study of Li-ion cells, J. Power Sources 101 (2001) 238247.
[18] Bork R et al 2011 New Control and Data Acquisition System in the Advanced LIGO Project, LIGO Document Control Center G1101141, available at https://dcc.ligo.org/public/0071/G1101141/001/

[19] Arduino micro-Controller. http://arduino.cc/

[20] Martin Nawrath: Frequency Measurement Library, available at http://interface.khm.de/index.php/lab/experiments/frequencymeasurement-library/

[21] Otto H. Schmitt, A Thermionic Trigger, Journal of Scientific Instruments 15 (January 1938): 2426.

[22] Agilent 33120A, Function/Arbitrary Waveform Generator, Data Sheet 2004, available at: http://cp.literature.agilent.com/litweb/pdf/59680125EN.pdf

[23] The Frequency Monitoring Network (FNET) project, http://powerit.utk.edu/fnet.html

[24] Norbert Wiener: "Extrapolation, Interpolation, and Smoothing of Stationary Time Series". 1949, New York: Wiley. ISBN 0-262-73005-7.

[25] Yutaka Ota et al: Autonomous distributed V2G (vehicle-to-grid) satisfying scheduled charging, IEEE Transactions on Smart Grid, vol. 3, no. 1, pp. 559564, 2012.

[26] Yutaka Ota et al: Autonomous Distributed Vehicle-to-Grid for Ubiquitous Power Grid and its Effect as a Spinning Reserve, The Journal of International Council on Electrical Engineering, 2011.4, pp. 214-221, 2011

[27] Yutaka Ota et al: Effect of Autonomous Distributed Vehicle-to-Grid on Power System Frequency Control, The Fifth IEEE International Conference on Industrial and Information Systems, Mangalore, India, July/August 2010

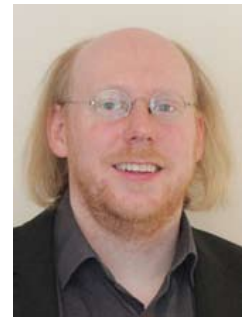

Stefan Hild received a physics degree (Dipl. Phys) from the University of Hannover, Germany, in 2003. In 2007 he received his $\mathrm{PhD}$ (Dr. rer. nat) from the Max-Planck Institute for Gravitational Physics (Albert-Einstein Institute) and the Leibniz University of Hannover for his work on the commissioning and enhancement of the British/German laser-interferometric gravitational wave detector GEO 600. After 2 years of postdoctoral research in Astrophysics and Space Research group of the University of Birmingham, UK, he obtained a faculty position at the University of Glasgow. Stefan Hild has a keen interest highprecision interferometry, quantum-non-demolition measurements as well as research into renewable energy and smart grid applications. He is the chair of the Sensing and Control Group of the GEO 600 collaboration.

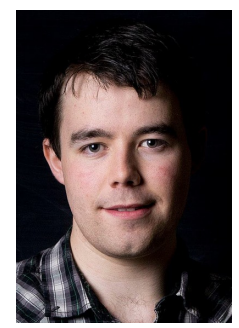

Sean Leavey received the MSci degree in physics from the University of Glasgow, United Kingdom, in 2012. He is currently undertaking a $\mathrm{PhD}$ degree at the Institute for Gravitational Research in the University of Glasgow, working on the instrumentation for ground-based, laser-interferometric gravitational wave detectors. Additionally, his research interests include the investigation of solutions for the problems facing future electricity grids.

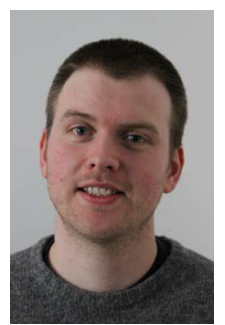

Christian Gräf received his Dipl. Phys. degree in physics from the Leibniz University of Hannover, Germany, in 2008. His current work focuses on optical design aspects of a prototype laser interferometer which aims for reaching and later surpassing the standard quantum limit for its 100 gram test masses. Besides laser interferometry his research interests include electronics and numerical optics. 


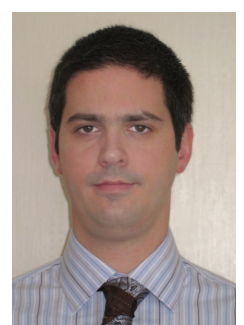

Borja Sorazu graduated in physics (specializing in electronic and control engineering) from the University of the Basque Country (UPV), Bizkaia, Spain, in 2000, and then he graduated in electronic engineering from the same university in 2001. Subsequently he received the Ph.D. degree in electronic and electrical engineering (specializing in optical fibre sensors and laser ultrasonics) from the University of Strathclyde, Glasgow, U.K., in 2006. During his Ph.D. he was involved in the use of optical techniques for structural examination of mechanical systems and material evaluation, including signal generation and acquisition and data interpretation. He has since joined the Institute for Gravitational Research (IGR) at the University of Glasgow, U.K., as a Research Associate, where his current research interest focus on several aspects of advanced interferometry for application to ground based gravitational wave detectors, and the detector characterization of GEO 600. 